\title{
Survey Data of Booster Magnet Misalignments
}

\author{
Sho Ohnuma*
}

July 31,2003

\section{Introduction}

As a part of the ongoing Booster improvement project, all the available data on booster magnets are collected and tabulated. Data include not only the survey results available at present (July 2003) but the magnet moves as well. The main purpose of this exercise is NOT to provide a basis for the future magnet moves. Rather, the goal is to present the present knowledge on where magnets are and incorporate it into, for example, the MAD lattice file so that one can see the impact of misaligned magnets on the closed orbit in the absence of any corrections.

Reference materials I have used for this task are:

1. Y.Chao, L.Ketcham, and C.Moore, "Understanding and Improving the Fermilab Booster High Field Orbit", TM-1571, March 1989.

2. Shekhar Shukla, "Preliminary Evaluation of the Survey Data collected in December 1993", informal note, January 25, 1994.

3. Carol Johnstone, “Booster 'As Built' Lattice”, BN-94-3, October 25, 1994.

4. Alex Waller, original survey data (private communication).

5. Jim Lackey, recorded moves of magnet from August 1994 to October 2002.

M. Popovic has done some preliminary processing of the survey data. I am grateful to him for giving me many valuable advices on this work. Jim Lackey has very patiently answered my numerous questions on all aspects of the magnet misalignment. It is hoped that this task will always be an integral part of the Booster Improvement Project.

\footnotetext{
* Affiliated with Department of Physics and Astronomy, University of Hawaii at Manoa, Honolulu, HI.
} 


\section{Survey Data}

Surveyors give three coordinates, $(\mathrm{X}, \mathrm{Y}, \mathrm{Z})$, of four reference holes $(\mathrm{A}, \mathrm{B}, \mathrm{C}, \mathrm{D})$ on top of each magnet. $A$ and $B$ are on the upstream side, $C$ and $D$ on the downstream side. $A$ and $\mathrm{D}$ are on the aisle side (radially outward), $\mathrm{B}$ and $\mathrm{C}$ on the wall side (radially inward). Looking from above, A-B-C-D is thus clockwise. What is not clear to me is the locations of these four reference holes relative to the "ideal" circular orbit in each magnet. This uncertainty creates a problem in specifying the radial offsets of each magnet.

$\mathrm{Z}$ is supposedly from the "sea level" but the data given to us by surveyors are in inches above 720 feet. A typical number is then 77.xxx. For some reason, which is not clear to me, the height $Z$ used by Booster Group (and in this work) is above $221.4325 \mathrm{~m}$ ( $=720$ feet $+1.9765 \mathrm{~m}$ ). For example, if $Z$ given by surveyors is 77.784 , it should be converted to -0.000786 (in meters).

$\mathrm{X}$ and $\mathrm{Y}$ are even more complicated. They are the original DUSAF coordinates (in feet) for which $A 0$ of the old main ring is $X=Y=100,000$. The site coordinates subtract this number so that $X=Y=0$ at $A 0$. The Booster Group apparently wanted to have $X$ and $Y$ of all magnets to be within $-100 \mathrm{~m}$ and $+100 \mathrm{~m}$. Instead of 100,000 feet $(=3,0480 \mathrm{~m})$, the offsets used are $3,0400 \mathrm{~m}$ for $\mathrm{X}$ and $3,0300 \mathrm{~m}$ for $\mathrm{Y}$. The direction of $\mathrm{Y}$-axis is still the original "Project North" of DUSAF and the X-axis is in the direction of "east". All of this is of course not essential to the relative location of each magnet, especially since it is quite unlikely that they would be surveyed again. The surveying that is currently going on is only for $Z$, the height of four reference holes.

Surveyors start numbering magnets from the first D-magnet after the long straight in each cell. On the other hand, the first magnet of each cell in MAD lattice file is the F-magnet immediately downstream of the short straight.

cell No. 1 F-D (Long Straight) D-F, cell No.2 F-D (Long Straight) D-F, etc in MAD lattice file.

With surveyors numbering, these are

cell No. $1 \quad 24-3,24-4,1-1,1-2$, (the last cell is cell No.24) cell No. $2 \quad 1-3,1-4,2-1,2-2$, etc. 
Four sets of coordinates $(\mathrm{X}, \mathrm{Y}, \mathrm{Z})$ for each magnet are given in a file called magnet_survey, http://www-ap.fnal.gov/ ohnuma/FNAL_03.dir/Booster_files.dir/ Coordinate values are in meters and the order is A-B-C-D.

\section{Vertical Misalignment}

For Z-values, the average, which is $-0.6872 \mathrm{~mm}$ at present, is already subtracted as one is interested in the relative height only. The average of $Z$ values will of course change as more magnets are resurveyed in the future. In the original table given to me by Alex Waller, -9999.0 is given for $\mathrm{Z}$ (and for $\mathrm{X}$ and $\mathrm{Y}$ as well) when there is no survey data available. I have supplied a "guessed" value using the convention $Z(A)=Z(D)$ and $Z(B)=Z(C)$. This implies that $I$ am ignoring the pitch and twist. For the roll, I use [<average of $Z(A)$ and $Z(D)>-<$ average of $Z(B)$ and $Z(C)>$ ] divided by the distance $0.38144 \mathrm{~m}$ between $\mathrm{A}$ and $\mathrm{B}$ (and between $\mathrm{C}$ and D which is also $0.38144 \mathrm{~m}$ ). Since MAD takes only one value of misalignment (dy for vertical position and dpsi for the roll) for each magnet, average of all four values of $Z$ is used for $\mathbf{d y}, \mathbf{d y}=$ average of $Z(A)$, $\mathrm{Z}(\mathrm{B}), \mathrm{Z}(\mathrm{C})$, and $\mathrm{Z}(\mathrm{D})$.

Although each magnet is represented by one value of $\mathbf{d y}$, the deviation from the average height, in MAD lattice file, mad_tab.out, it may be necessary to know dy at the upstream and the downstream ends separately, especially for a possible magnet alignment. This information is available in heightroll_tab where magnets are listed in the order specified in MAD lattice file. In terms of surveyor's numbering,

$$
1=24-3, \quad 2=24-4, \quad 3=1-1,4=1-2, \ldots .95=24-1,96=24-2 .
$$

Units are meters for $\mathbf{d y}$ and radians for dpsi.

\section{Horizontal Misalignment}

The following statement is from Ref. 1, TM-1571, which is still very much relevant in discussing the horizontal misalignment of Booster magnets.

"The question of determining the absolute horizontal beam trajectory of a circular machine is not a trivial one. This is due to the difficulty in coordinating the 
measurements on widely different objects such as the magnetic field, the effective magnetic length, and the positions of all relevant elements. The cross influence between the two planes adds to the complexity. These factors are further obscured by the flexibility demanded by beam control such as low energy guide field and radial feedback loops. This ambiguity is then propagated to the alignment errors which are measured against the "ideal" beam trajectory."

The report then explains how the radial offsets of each magnet have been obtained from the survey data on $(X, Y)$. Similar explanations are given in Refs. 2 and 3, albeit rather sketchy. The difficulty of referencing the actual magnet positions is obvious when one finds many discrepancies between Ref. 2 and Ref. 3. They are issued in the same year (January and October of 1994) and, according to Jim Lackey, no magnet was moved horizontally during that year. It is more difficult to understand the significant discrepancies between results in Fig. 8 of Ref. 2, called r-disp (obs-ideal), and the corresponding figures given to me recently by Jim Lackey. The latter includes all the moves made from December '97. For example, the average radial displacement of magnets $13-1$ to $18-4$ is $-0.72 \mathrm{~mm}$ in Ref. 2 while it is $+8.45 \mathrm{~mm}$ in Lackey's figure. Magnet moves recorded by Jim Lackey cannot explain this large difference.

In view of this difficulty, I have decided to forget the so-called "ideal magnet locations" altogether and simply record the radial position of each magnet relative to a suitable average value. I say "suitable" since the average distances from the ring center to F- and D-magnets are not the same. Each magnet position is represented by the average $(\mathrm{X}, \mathrm{Y})$ of four reference holes. Since the positions of these four holes relative to the ideal horizontal orbit in a magnet are unknown (to me), I am not sure if these offsets would help understanding the closed orbit behavior. One thing is clear to me, though. It would be extremely dangerous to move a magnet radially based solely on its location relative to the "ideal" location, however one defined the "ideal".

Radial offset of each magnet center is given in mad_tab.out as dx. Another file called r_position_tab lists the offsets separately for upstream and downstream. Although this file may be used as a basis for possible magnet moves, moving a magnet radially (in contrast to moving vertically) should be done only when one knows what one is doing. Altogether ten different average values are used ("suitable") to find the offsets: upstream 
and downstream of two types (the first and the last in each cell) of F-magnets and two types of D-magnets, and the centers of F-magnets and of D-magnets.

In the following, steps taken for finding the offsets of each magnet (upstream, downstream, and center) are explained. It is not claimed here that these are the best procedures. Rather, the explanation would make clear of what I have done. Others may disagree and use different procedures to find the offsets.

1. In the original survey data, eighteen magnets do not have the complete set (four) of $(X, Y)$. They are

2-4(C) 3-1(B) 3-2(C) 3-3(B) $\quad 13-1(\mathrm{~B} \& \mathrm{C}) \quad$ 13-2(B\&C) $\quad 13-3(\mathrm{~A})$

13-4(C) 14-4(C) 15-4(C) $16-4$ (C) $17-3$ (B) $18-4$ (C) 20-4(C) $21-4(\mathrm{C})$

$22-4(\mathrm{C}) \quad 23-4(\mathrm{C}) \cdot 24-4(\mathrm{C})$

When only one hole is missing, for example $\mathrm{C},(\mathrm{X}, \mathrm{Y})$ of three other holes are used together with the known average of three distances, for example CA, CB, and $C D$, to supply the missing values. Two exceptions to this are magnets 3-1 and 3-3 for which no consistent figures can be found. Together with magnets 131 and 13-2 where $(\mathrm{X}, \mathrm{Y})$ of two holes are missing, there are four magnets without four complete sets of $(X, Y)$.

2. When all four sets of $(X, Y)$ are available, the center coordinates of each magnet are found from the average of all four sets.

3. By taking pairs such as 1-1 and 13-1, 1-2 and 13-2, etc., the ring center coordinates (Xc, Yc) are found. This is not unique since each pair gives a slightly different ring center. An approximate center is defined to be the average of all.

4. Distances to all F-magnets, $r_{f}(k)$, and to D-magnets, $r_{d}(k)$, from the center are calculated and they are used to find the average values $r_{f}(a v)$ and $r_{d}(a v)$.

5. The final values of $(\mathrm{Xc}, \mathrm{Yc}$ ) are found by minimizing the sum of all available values of $\left[r_{f}(k)-r_{f}(a v)\right]^{2}$ and $\left[r_{d}(k)-r_{d}(a v)\right]^{2}$. This is (almost) equivalent to filtering out the first harmonic content of $r_{f}(k)$ and $r_{d}(k)$ in the entire ring. 
These procedures lead to the following results:

$$
(\mathrm{Xc}, \mathrm{Yc})=-3.122863 \mathrm{~m} \text { and } 6.536856 \mathrm{~m} \text {. }
$$

Average distance:

Upstream Downstream Center

$\begin{array}{llll}\text { First F } & 75.528077 & 75.503293 & 75.501628 \\ \text { First D } & 75.491016 & 75.406812 & 75.434851 \\ \text { Second D } & 75.406788 & 75.491116 & 75.434901 \\ \text { Second F } & 75.503446 & 75.527995 & 75.501662\end{array}$

These numbers are all in meters and their rms values are typically $2 \mathrm{~mm}$. For $\mathbf{d x}$ in mad_tab.out, the average distance of all F-magnets (first and second) is taken to be $75.501645 \mathrm{~m}$. For all D-magnets, it is $75.434875 \mathrm{~m}$. Offsets in $\mathbf{r} \_$position_tab use eight different average values since their differences are an order of magnitude larger than their rms values.

\section{Misalignment of Beam Position Monitors (BPM)}

In order to see the impact of magnet misalignments on the closed orbit, it is necessary to know the misalignment of each BPM although there are no direct survey data. The particular version of MAD, MAD-8, which is currently used at Fermilab by most people, cannot handle the effect of misaligned BPM. That is, if the closed orbit is 0 before a BPM is misaligned, it will be shown as 0 even after a misalignment, this in spite of the fact that the manual clearly explains how to incorporate the effect. [See p. 74 and 75 of the MAD manual for Version 8.13, issued on January 10, 1994.] I have asked Hans Grote and Frank Schmidt at CERN but I do not have any answer to this question. When the magnet misalignment information (mad_tab.out) is included in MAD lattice file, the closed orbit at BPMs shown in MAD output files must be modified by the BPM misalignment. BPM misalignments are listed in monitor_xtab for horizontal BPMs and 
in monitor_ztab for vertical BPMs. Numbers for $\mathbf{d x}$ and $\mathbf{d y}$ are all in meters and these numbers should be subtracted from the MAD output results in order to compare with the actual readings of BPMs. For example, in monitor_xtab, one sees $\mathbf{d x}=-0.003476$ for HP05S. If MAD output says the horizontal closed orbit at this BPM is $+3.2 \mathrm{~mm}$, the real $B P M$ reading should be $+3.2 \mathrm{~mm}-(-3.476) \mathrm{mm}=+6.676 \mathrm{~mm}$.

Since the precise radial and vertical positions of BPMs relative to the magnet positions are not known, the following assumptions are used in preparing monitor_xtab and monitor_ztab following a suggestion from Jim Lackey. For monitors at the middle of each short straight section, the average position of the downstream end of upstream magnet and the upstream end of the downstream magnet (both magnets are F) is used. For example, for $\mathbf{d x}$ of monitor HP07S, the relevant magnets are 7-2 (upstream) and 7-3 (downstream). For monitors near the downstream end of each long straight, the upstream position of the nearest magnet (always D) is used directly. Again it should be cautioned that this may not reflect the true position of every BPM but, in the absence of better information, the choice is not unreasonable.

\section{Concluding Remarks}

All future survey results (vertical) and magnet moves should be carefully recorded and incorporated in the appropriate files so that these files are up-to-date all the time. Processing of these new data, either following the procedures described in this note or something even better, will be required to make the information useful in the understanding of the Booster orbit. 